\title{
Salicylate toxicity model of tinnitus
}

\section{Daniel Stolzberg *, Richard J. Salvi and Brian L. Allman}

Center for Hearing and Deafness, University at Buffalo, The State University of New York, Buffalo, NY, USA

\section{Edited by:}

Jos J. Eggermont, University of Calgary, Canada

Reviewed by:

Arnaud Norena, Université de

Provence, France

Ana Elgoyhen, INGEBI, Argentina

*Correspondence:

Daniel Stolzberg, Center for Hearing and Deafness, University at Buffalo,

The State University of New York,

137 Cary Hall, Buffalo, NY 14214,

USA.

e-mail:djs32@buffalo.edu
Salicylate, the active component of the common drug aspirin, has mild analgesic, antipyretic, and anti-inflammatory effects at moderate doses. At higher doses, however, salicylate temporarily induces moderate hearing loss and the perception of a high-pitch ringing in humans and animals. This phantom perception of sound known as tinnitus is qualitatively similar to the persistent subjective tinnitus induced by high-level noise exposure, ototoxic drugs, or aging, which affects $\sim 14 \%$ of the general population. For over a quarter century, auditory scientists have used the salicylate toxicity model to investigate candidate biochemical and neurophysiological mechanisms underlying phantom sound perception. In this review, we summarize some of the intriguing biochemical and physiological effects associated with salicylate-induced tinnitus, some of which occur in the periphery and others in the central nervous system. The relevance and general utility of the salicylate toxicity model in understanding phantom sound perception in general are discussed.

\section{Keywords: salicylate, aspirin, tinnitus, hearing loss, auditory dysfunction, animal models}

\section{INTRODUCTION}

Subjective tinnitus is characterized by the perception of a sound in the absence of an acoustic source in the environment. A recent health census in the United States indicated approximately 50 million adults report having experienced tinnitus and 16 million experience it frequently (Shargorodsky et al., 2010).

Although the cochlea is responsible for transducing acoustic signals into neural activity, it is becoming clear that the brain is capable of generating neural activity of its own within the auditory pathway leading to tinnitus perception. Subjective tinnitus is primarily associated with sensorineural hearing loss (Norena et al., 2002; Weisz et al., 2006) which may be the consequence of loud noises, ototoxic drugs, or aging. While noise exposure is likely the most common trigger of tinnitus (Axelsson and Sandh, 1985), several ototoxic drugs are known to cause tinnitus in humans and animals. Among these are the chemotherapeutics such as cisplatin (Bokemeyer et al., 1998; Rachel et al., 2002), the antimalarial drug quinine (Kenmochi and Eggermont, 1997; Ochi and Eggermont, 1997; Eggermont and Kenmochi, 1998; Lobarinas et al., 2006; Ralli et al., 2010), and salicylate, the active component of the non-steroidal anti-inflammatory drug aspirin (Cazals, 2000). At therapeutic doses salicylate has mild analgesic, anti-inflammatory, and antipyretic effects and is commonly used for minor headaches and traditionally for rheumatic arthritis. At high doses, however, salicylate causes temporary hearing loss and reversible tinnitus, while hyperventilation, vertigo, confusion, hemorrhaging, or death may result from extremely high doses (Graham and Parker, 1948; Rauschka et al., 2007).

Since the establishment of a behavioral model of salicylateinduced tinnitus in rats (Jastreboff et al., 1988a,b), the drug has been used in various animal species to investigate the biological mechanisms of tinnitus generation. Although the mechanisms of tinnitus induction by salicylate may differ from those caused by other hearing disorders, salicylate-induced tinnitus has several distinct advantages over other methods of tinnitus induction such as acute noise trauma. Two such advantages are the rapid induction and reversibility of salicylate-induced tinnitus. Following oral consumption or systemic injection of the drug, tinnitus presents within minutes and subsides within $72 \mathrm{~h}$ of the final dose (Mongan et al., 1973). Interestingly, in a study by Mongan et al. (1973), it was found that the majority of humans with preexisting hearing loss $(\sim 68 \%)$ did not report tinnitus despite very high blood serum levels of salicylate. In rats, induction of tinnitus by systemic administration of salicylate was found to be highly reliable with proper dosing (Lobarinas et al., 2006), whereas noise trauma has a much more variable success of tinnitus induction (Kraus et al., 2010).

Salicylate has several well-established effects on cochlear function; however, more recent experiments have highlighted the drug's direct modulation of neural activity in the brain. The cochlear and central effects of salicylate should come as no surprise since the drug rapidly enters cochlear perilymph and cerebrospinal fluid simultaneously (Jastreboff et al., 1986). The widespread distribution of the drug adds to the complexity of understanding the mechanisms that are directly responsible for salicylate-induced tinnitus. Here we review some critical features of the salicylate toxicity model of tinnitus. We begin with a discussion of salicylate's primary effects in the cochlea, followed by its modulation of neurotransmitter systems and neural activity within the brain. Insights gained from the salicylate model of tinnitus continue to inform researchers about the potential mechanisms that lead to tinnitus perception.

\section{COCHLEAR EFFECTS}

Salicylate ototoxicity differs from most ototoxic drugs in that hearing loss and tinnitus normally subside within 1-3 days following cessation of treatment (Myers and Bernstein, 1965). Several independent effects of salicylate on the peripheral 
auditory system have been identified, each of which likely contribute to some aspect of the resulting hearing loss (summarized in Figure 1). Furthermore, salicylate-induced tinnitus has been hypothesized by some groups to be generated by altered cochlear activity and transmitted with fidelity to the central auditory system.

There exists a strong linear correlation between plasma levels of unbound salicylate and the extent of decreased auditory sensitivity (Day et al., 1989) reaching asymptote at a threshold of $\sim 40 \mathrm{~dB}$ elevation despite increased dosing (Myers and Bernstein, 1965; McFadden and Plattsmier, 1984); however, the relationship of blood plasma salicylate levels to the presence of tinnitus is much less predictable (Mongan et al., 1973; Halla et al., 1991). This threshold shift can be accounted for by dysfunction of the outer hair cell (OHC) active response to sound. Otoacoustic emissions (OAEs) are acoustic signals generated by the electromotile $\mathrm{OHCs}$ and serve to improve sensitivity and sharpen frequency selectivity. Sound evoked OAEs, especially distortion product OAEs (DPOAE), are reduced in humans and animals at low to moderate stimulus levels during salicylate toxicity (Wier et al., 1988; Kujawa et al., 1992; Fitzgerald et al., 1993; Guitton et al., 2003; Ruel et al., 2008; Ralli et al., 2010; Stolzberg et al., 2011). In vitro, salicylate alters membrane conductance and the shape of OHCs (Douek et al., 1983; Shehata et al., 1991). Subsequent experiments showed that salicylate acts as a competitive antagonist for the chloride anion binding site of prestin (Oliver et al., 2001), the motor protein of OHCs, resulting in inhibition of OHC motility (Kakehata and Santos-Sacchi, 1996; Zheng et al., 2000).

Recently, we reported that acute systemic salicylate treatment in ketamine/xylazine anesthetized rats $(300 \mathrm{mg} / \mathrm{kg}$ sodium salicylate, i.p.) suppresses high and low frequency DPOAEs to a greater extent than at middle frequencies (Stolzberg et al., 2011). Interestingly, the region of greatest sensitivity following salicylate corresponded to the behaviorally estimated pitch of tinnitus in rats (Yang et al., 2007; Kizawa et al., 2010).

The active generation of a sound within the cochlea by spontaneous OAEs (SOAEs) was an early hypothesis pointing to a peripheral generator of tinnitus (Wilson, 1980). SOAEs can be measured in $\sim 38 \%$ of the population (Wier et al., 1984); however, most people with SOAEs do not have tinnitus. Conversely, SOAEs are absent in many people with tinnitus (Zurek, 1981). Salicylate treatment reduces or abolishes SOAEs in humans (McFadden and Plattsmier, 1984; Wier et al., 1988). Thus, SOAEs are unlikely to be a generator of the tinnitus signal.

Other measures of cochlear function, such as the cochlear microphonic (CM) and summating potential (SP), have been evaluated following local or systemic salicylate administration. The CM is an electrical signal that reflects the net flow of ionic currents alternating across cellular membranes in response to a periodic sound stimulus such as a tone. The CM, therefore, provides a gross index of the permeability of primarily OHCs of the inner ear. The CM has been shown to be unaffected in guinea pigs following cochlear perfusion of salicylate in response to $10 \mathrm{kHz}$ tones (Puel et al., 1990), but increased in response to $1 \mathrm{kHz}$ tones (Fitzgerald et al., 1993). Fitzgerald et al. (1993) attributed the different results of the two studies to the choice

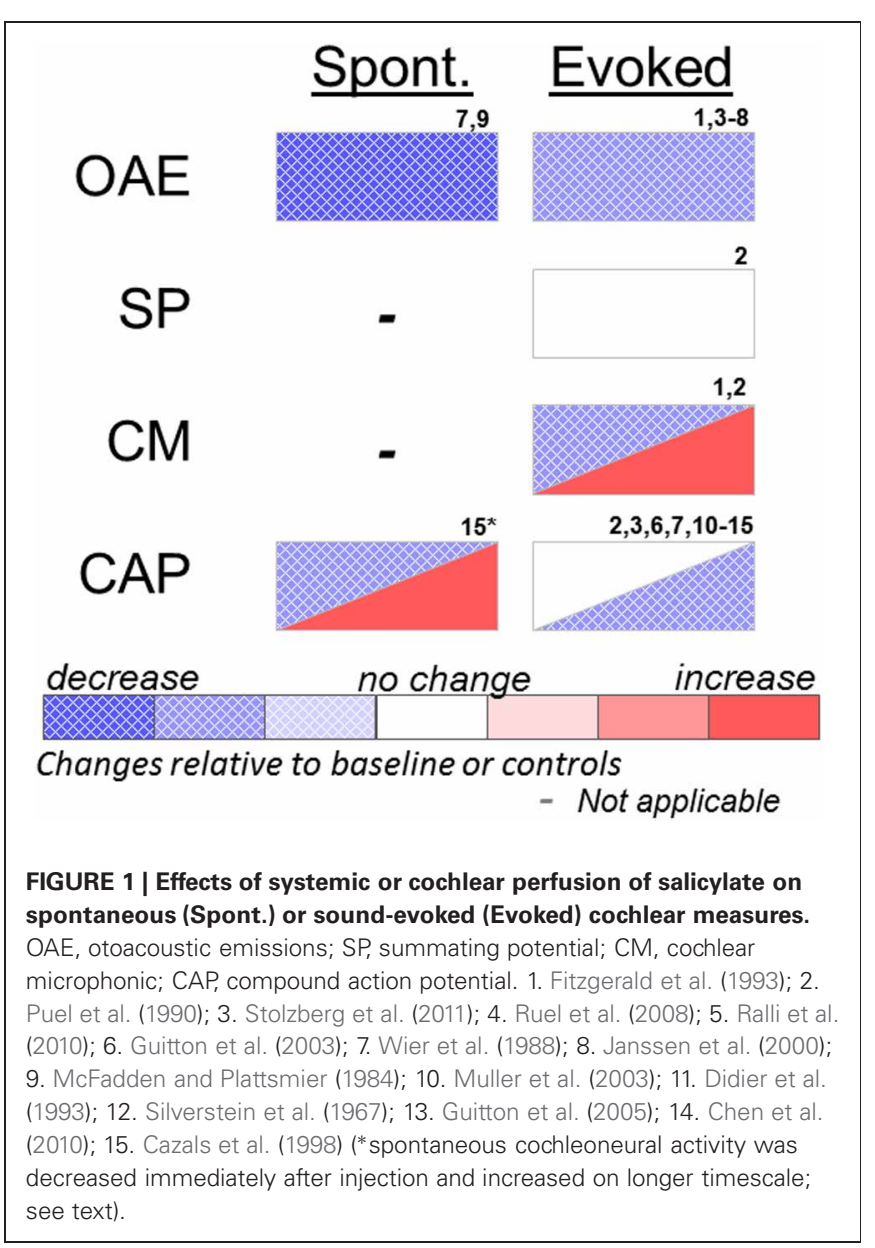

of stimulus frequency. The SP is a measure of the direct-current response of hair cells to sound recorded from the round window of the cochlea and most likely reflects both IHC and OHC function (Durrant et al., 1998). Cochlear perfusion of salicylate did not have an effect on SP in guinea pigs (Puel et al., 1990).

Auditory nerve (AN) fiber recordings following systemic salicylate administration have yielded variable results, most likely attributable to differential effects of moderate versus high dosing levels. In cats, Evans and Borerwe (1982) observed a significant increase in spontaneous firing rates following a high dose of sodium salicylate $(400 \mathrm{mg} / \mathrm{kg}$ i.v. $)$ in a subpopulation of AN fibers with baseline high spontaneous rate. This dose is extremely high for the cat since a slightly lower dose $(350 \mathrm{mg} / \mathrm{kg}$ i.p. $)$ has been reported to result in hyperventilation and death (Silverstein et al., 1967). In contrast, AN fibers in gerbils showed a small, yet significant, decrease in spontaneous firing rates in fibers with low characteristic frequencies (CFs) and no change in fibers with high CFs following a moderate dose of salicylate $(200 \mathrm{mg} / \mathrm{kg}$ i.p. salicylic acid) (Muller et al., 2003). Kumagai (1992) reported that a high systemic dose $(400 \mathrm{mg} / \mathrm{kg}$ i.v. sodium salicylate) significantly increased AN spontaneous firing rates in guinea pigs, whereas spontaneous firing rates were not affected by the low systemic dose ( $200 \mathrm{mg} / \mathrm{kg}$ i.v. sodium salicylate). The lower dose of salicylate, although it did not alter spontaneous activity in 
guinea pig AN, is sufficient to induce tinnitus-like behavior in rats (Lobarinas et al., 2006).

The effects of long-term salicylate treatment on spontaneous AN activity have also been investigated for changes in the average spectrum of electrophysiological cochleoneural activity (ASECA) recorded from a round window of guinea pigs (Cazals et al., 1998). The magnitude of the ASECA measure reflects spontaneous activity of the AN. ASECA decreased following the initial treatment of salicylate, but increased from baseline levels after approximately 1 week of treatments $(200 \mathrm{mg} / \mathrm{kg}$ sodium salicylate, i.m., twice per diem). The results from this study indicate that acute dosing reduces spontaneous AN activity, whereas long-term dosing increases spontaneous AN activity.

Recently, research has explored the mechanisms of increased spontaneous AN fiber activity at very high doses of salicylate. Salicylate confers many of its therapeutic effects through inhibition of the inducible form of cyclooxygenase, COX-2, an important enzyme in the anti-inflammatory response of cells (Vane, 1998). The intracellular fatty acid, arachidonic acid, is a second messenger that is cleaved by the COX-2 enzyme, resulting in the synthesis of prostaglandins. The presence of arachidonic acid in high concentrations is known to potentiate $N$-Methyl$\mathrm{D}$-aspartate (NMDA) ionic currents in spiral ganglion neurons (Miller et al., 1992). Peng et al. (2003) showed salicylate does indeed potentiate NMDA currents in isolated pre-hearing postnatal mouse spiral ganglion cells. Ruel et al. (2008) further demonstrated that salicylate potentiated NMDA currents in AN afferent terminals by increasing available arachidonic acid. In addition, perilymphatic perfusion of a high concentration of salicylate (5 mM) increased AN spontaneous firing rates (Ruel et al., 2008). The same group (Guitton et al., 2005) demonstrated that blockade of NMDA channels prevented rats from developing salicylateinduced tinnitus, supporting their hypothesis that tinnitus is generated within the cochlea. In the study by Ruel et al. (2008), increased spontaneous firing rates of AN fibers was proposed to underlie tinnitus generation; however, the NMDA currents driving increased AN firing rates may only occur at extremely high concentrations of salicylate, far above those sufficient to induce tinnitus. In fact, the $5 \mathrm{mM}$ sodium salicylate used in this study was the peak concentration measured in guinea pig perilymph following systemic treatment with $460 \mathrm{mg} / \mathrm{kg}$ (Jastreboff et al., 1986), a dose well above that sufficient to induce behavioral evidence of tinnitus in animals (Lobarinas et al., 2006). Indeed, Ruel et al. (2008) indicated in their report that $1 \mathrm{mM}$ sodium salicylate failed to elicit detectable NMDA currents in spiral ganglion neurons. Thus, some of the reported effects of salicylate in the cochlea likely occur only at very high concentrations and may not be directly involved in tinnitus generation per se, but may play a role in determining the quality of the tinnitus percept. Anecdotal evidence suggests that at extremely high dose levels of aspirin alters the perception of tinnitus from reasonably tonal to cricketlike (see discussion in McFadden et al., 1984). It is plausible that a cricket-like perception may result from increased spontaneous AN activity.

Overall, salicylate's effects in the cochlea following systemic administration manifest as a reduction of sound-driven activity, primarily due to the reduction of $\mathrm{OHC}$ electromotility.
Furthermore, at doses sufficient to induce tinnitus in animals, salicylate does not seem to significantly increase spontaneous AN activity. Taken together, the evidence from the literature seems to argue against AN hyperactivity as the direct cause of tinnitus; therefore, the cochlea is not likely generating the tinnitus signal. Moreover, a purely cochlear model of tinnitus generation is problematic because spontaneous activity is always present in the AN (Walsh et al., 1972) but does not normally cause tinnitus perception. While spontaneous AN activity is important for the encoding of acoustic signals (Koerber et al., 1966; Liberman and Kiang, 1978), the brain is normally capable of tuning out peripheral spontaneous activity unrelated to acoustic stimulation; i.e., most people do not experience tinnitus despite having spontaneous AN activity. Based on anatomical and electrophysiological studies of the brain in animal models of tinnitus, the following sections provide compelling evidence for a central contribution to salicylate-induced phantom sound perception.

\section{CENTRAL EFFECTS}

Salicylate clearly decreases the sensitivity of the sensory epithelium to sound and may modulate the rate of spontaneous AN transmission; however, there is also strong evidence that the drug directly modulates neurotransmission in the brain. An early study in human subjects provided evidence that the perception of tinnitus most often precedes self-reported hearing loss (Mongan et al., 1973). Since salicylate is delivered to both the perilymph and cerebrospinal fluid (Jastreboff et al., 1986), the report that tinnitus perception precedes self-reported hearing loss may indicate that the brain is more sensitive to the presence of salicylate than the cochlea. Furthermore, as discussed below, salicylate has been found to directly modulate inhibitory neurotransmission in the brain which may facilitate the rapidity at which phantom sound is experienced following systemic administration of the drug. The decreased cochlear sensitivity and/or alterations in spontaneous firing rates in the AN may serve more to determine the tinnitus pitch, whereas salicylate's direct effects on the brain act to generate and/or permit spurious neural activity to reach awareness.

\section{IDENTIFYING KEY BRAIN REGIONS}

Cytological markers of neural activity have been observed in several auditory and non-auditory brain regions in salicylateinduced tinnitus (Figure 2). The earliest studies used radioactive glucose, $\left[{ }^{14} \mathrm{C}\right] 2$-deoxyglucose (2-DG) (Kauer et al., 1982; Wallhausser-Franke et al., 1996); whereas more recent studies have utilized immunolabeling of the immediate early gene $c$-fos (Wallhausser-Franke, 1997; Wallhausser-Franke et al., 2003; Wu et al., 2003), which is up-regulated in response to increased neural activity. Chronic systemic salicylate treatment at a dose known to induce behavioral evidence of tinnitus in gerbils resulted in reduced 2-DG uptake in the inferior colliculus (IC), particularly in the high-frequency responsive region, while increased uptake was observed in the auditory cortex (AC), as compared to saline controls (Wallhausser-Franke et al., 1996). In subsequent studies, small increases in c-fos were observed following acute systemic salicylate treatment $(350 \mathrm{mg} / \mathrm{kg}$ i.p., sufficient to induce tinnitus) in the dorsal cochlear nucleus $(\mathrm{dCN})$ and the dorsal division of the medial geniculate body (dMGB), but not in ventral 


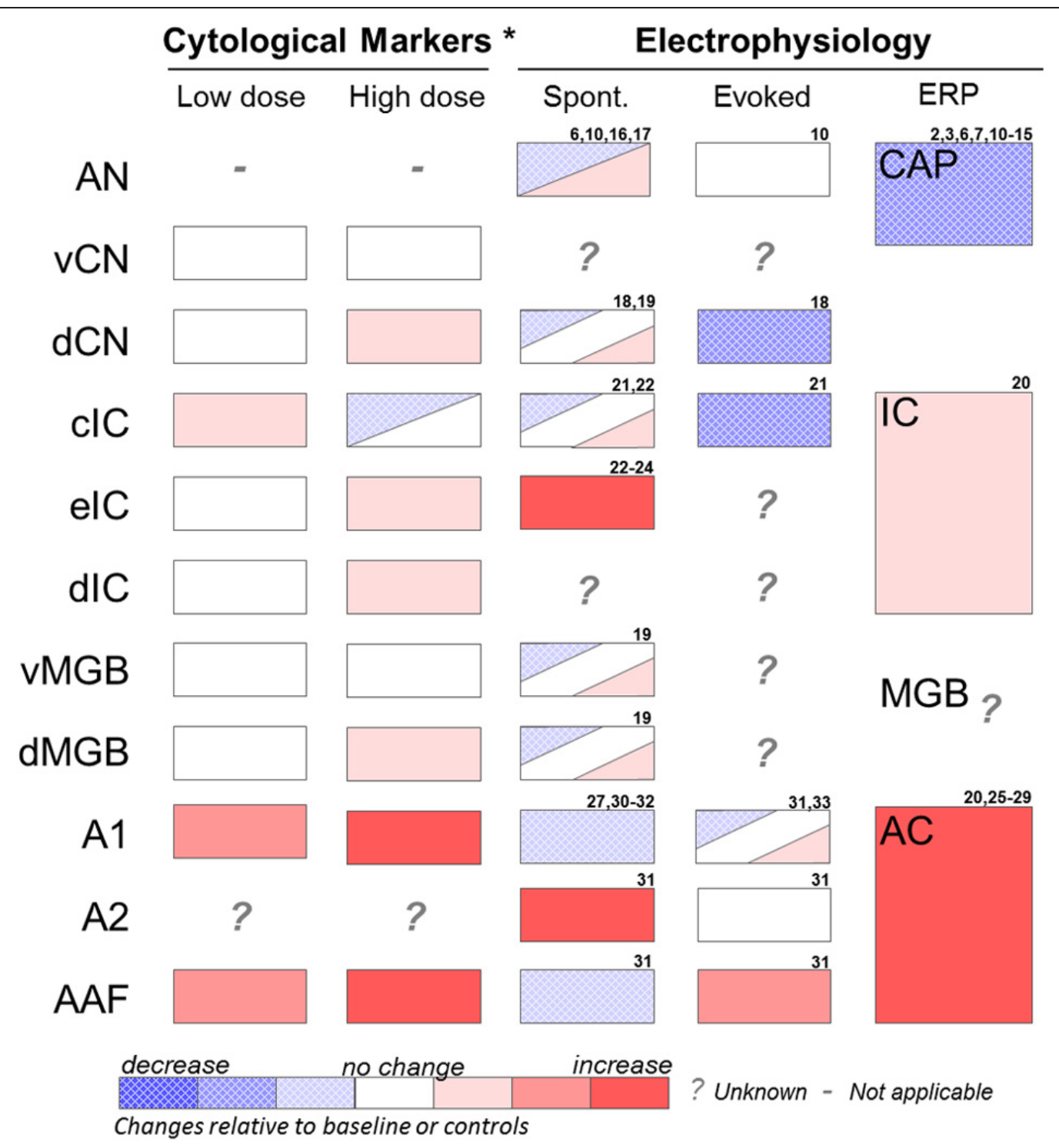

FIGURE 2 | Summary of effects of salicylate on various auditory structures. Cytological studies include results from 2-DG and fos-IR imaging studies (see Section "Identifying Key Brain Regions") and were performed at low (50 mg/kg i.p.) or high (350 mg/kg i.p.) acute doses of salicylate. Electrophysiology recordings were performed at various moderate to high salicylate doses. Results are from studies using in vivo extracellular recordings or in vitro extracellular or patch clamp recordings (see Section "Electrophysiology"). AN, auditory nerve; vCN, ventral cochlear nucleus; $\mathrm{dCN}$, dorsal cochlear nucleus; $\mathrm{ClC}$, central nucleus of the inferior colliculus; elC, external nucleus of the inferior colliculus; dIC, dorsal nucleus of the inferior colliculus; VMGB, ventral portion of the medial geniculate body; dMGB, dorsal portion of the medial geniculate body; $A 1$, primary auditory cortex; A2, secondary auditory cortex; AAF, anterior auditory field. Spont, single unit or multiunit spontaneous firing rates; Evoked, sound-evoked single unit or multiunit firing rates; ERP, sound-evoked response field potential;
CAP, compound action potential of the cochlea; IC, inferior colliculus response potential; MGB, medial geniculate response potential; AC, auditory cortex response potential. *Represents acute effects of salicylate treatment (Wallhausser-Franke, 1997; Wallhausser-Franke et al., 2003). 2. Puel et al. (1990); 3. Stolzberg et al. (2011); 6. Guitton et al. (2003); 7. Wier et al. (1988); 8. Janssen et al. (2000); 9. McFadden and Plattsmier (1984); 10. Muller et al. (2003); 11. Didier et al. (1993); 12. Silverstein et al. (1967); 13. Guitton et al. (2005); 14. Chen et al. (2010); 15. Cazals et al. (1998); 16. Evans and Borerwe (1982); 17. Kumagai (1992); 18. Wei et al. (2010); 19. Basta et al. (2008); 20. Sun et al. (2009); 21. Ma et al. (2006); 22. Basta and Ernst (2004); 23. Jastreboff and Sasaki (1986); 24. Chen and Jastreboff (1995); 25. Lobarinas et al. (2006); 26. Norena et al. (2010); 27. Lu et al. (2011); 28. Lobarinas et al. (2006); 29. Paul et al. (2009); 30. Yang et al. (2007); 31. Eggermont and Kenmochi (1998); 32. Kenmochi and Eggermont (1997); 33. Zhang et al. (2011).
CN (vCN) or ventral MGB (vMGB) (Wallhausser-Franke, 1997; Wallhausser-Franke et al., 2003). The largest increases in c-fos expressing cells following salicylate (eight times the number of cells than control) were observed in AC, especially in primary $\mathrm{AC}$
(A1) and anterior auditory field (AAF) (Wallhausser-Franke et al., 2003). In contrast, the central nucleus of the IC (cIC) was virtually free of c-fos and only a moderate increase was observed in dorsomedial IC (dIC) (Wallhausser-Franke, 1997), which receives 
descending fibers from $\mathrm{AC}$ and does not belong to the ascending auditory pathway (Faye-Lund, 1985). Chronic dosing of salicylate in rats showed similar results for the $\mathrm{CN}$ and $\mathrm{dIC}$, but differed in that increased c-fos was additionally observed in cIC (Wu et al., 2003). Interestingly, four times the number of cells expressing c-fos was observed in AC following systemic treatment of salicylate at a low dose $(50 \mathrm{mg} / \mathrm{kg})$, which is insufficient to induce behavioral evidence of tinnitus (Wallhausser-Franke et al., 2003). In the same study, a faint increase in c-fos expression was also observed in IC following the low, but not the high dose of salicylate. These results indicate that salicylate has effects on neural activity in the brain even at sub-tinnitus-inducing doses, indicating that the brain, and in particular $\mathrm{AC}$, is highly sensitive to the presence of salicylate.

Paul and colleagues (2009) used a metabolic tracer, fluorine-18 deoxyglucose (FDG), and positron imaging tomography (PET) to image activity in the entire rat brain following a single dose of sodium salicylate $(250 \mathrm{mg} / \mathrm{kg}$ i.p.). Metabolic activity was significantly increased in IC and AC; a small trend toward an increase was measured in the thalamus, but this did not reach statistical significance. The greatest increase in metabolic demands was observed in AC, followed by the IC, corroborating the results from immunocytochemistry experiments described above.

Holt and colleagues investigated the effects of chronic salicylate ( 3 consecutive days at $300 \mathrm{mg} / \mathrm{kg}$ i.p) in rats using manganeseenhanced MRI, a technique that assesses brain activity based on the uptake of manganese into active neurons (Holt et al., 2010). This technique, which has substantially higher spatial resolution than PET, measured significantly increased signal strength in $\mathrm{dCN}$, but not in $\mathrm{vCN}$, following chronic salicylate treatment, supporting the results of earlier $c$-fos studies. A significant increase in signal strength in $\mathrm{CIC}$, dIC, and external nucleus of the IC (eIC) are consistent with earlier cytological studies after chronic salicylate dosing protocol (Wallhausser-Franke et al., 1996; Wu et al., 2003). Interestingly, this MRI study did not observe any significant changes in AC following salicylate treatment, whereas previous 2-DG studies with a similar dosing protocol had observed significant c-fos and metabolic activity in AC (Wallhausser-Franke et al., 1996; Paul et al., 2009).

Wallhäuser-Franke concluded: "If tinnitus is evoked by the effects of salicylate on the cochlea, increased c-fos immunoreactivity would be expected in auditory brainstem nuclei, especially since auditory stimulation readily evokes c-fos expression, for example in IC (see discussion in Wallhausser-Franke, 1997)." Following acoustic stimulation, the lemniscal auditory pathway structures (vCN, cIC, vMGB, and A1) all exhibit elevated c-fos expression (Carretta et al., 1999). As discussed in the previous section (see Section "Cochlear Effects"), if acute salicylate treatment does indeed increase spontaneous firing rates of AN fibers (Evans and Borerwe, 1982; Ruel et al., 2008) then 2-DG uptake and c-fos would be expected to be enhanced in the $\mathrm{vCN}$, cIC, and vMGB. The apparent lack of c-fos within the lemniscal auditory structures following acute dosing of salicylate suggests that tinnitus-related hyperactivity originates within the brain and is related primarily to the AC and extralemniscal midbrain and brainstem structures (dMGB, dIC, and dCN). Results from immunocytochemistry studies are, therefore, more in line with the observations of reduced AN spontaneous firing rates recorded in gerbils following moderate systemic doses of salicylate sufficient to induce tinnitus (Muller et al., 2003).

While cytological studies support the hypothesis that central auditory structures play a critical role in tinnitus generation, it remains unclear if the hyperactivity in these auditory structures is the result of direct action of the drug on neural activity, the indirect result of dynamic adjustments of neural networks to decreased peripheral output, or a complex interaction between these peripheral and central effects. Furthermore, it should be noted that although this review is focused on alterations along the classical auditory pathway following salicylate, effects have been observed in other brain regions, such as limbic structures, and may play important roles in tinnitus (Wallhausser-Franke, 1997). In the following subsections, we will discuss evidence that salicylate does indeed directly modulate neurotransmission in the brain.

\section{EFFECTS ON GABAergic NEUROTRANSMISSION}

Salicylate has been shown to directly affect neurotransmitter systems in the brain. One important factor in salicylate-induced tinnitus is the drug's apparent direct actions on $\gamma$-aminobutyric acid (GABA) inhibitory neurotransmission in the brain.

Chronic treatment with salicylate in rats resulted in the increased expression of glutamic acid decarboxylase $\left(\mathrm{GAD}_{65}\right)$, the GABA synthesizing enzyme, in IC (Bauer et al., 2000). The increase in $\mathrm{GAD}$ was postulated to be compensatory for increased neural traffic. In the same study, a significant increase in $\mathrm{GABA}_{A}$ receptor affinity in $\mathrm{cIC}$, eIC, and $\mathrm{dIC}$ was measured. These changes may be the result of increased neural activity, direct effects of salicylate on GAD activity, or both. Indeed, changes in $\mathrm{GAD}$ concentration following salicylate treatment may be the result of direct inhibition of GAD enzymatic activity by salicylate (Gould et al., 1963; Gould and Smith, 1965); however, these effects were measured at relatively high concentrations in vitro.

Functional changes of GABAergic interneurons in the auditory system have also been observed. Salicylate applied to brain slices $(1.4 \mathrm{mM})$ significantly decreased the frequency and amplitude of current-evoked and spontaneous-miniature inhibitory postsynaptic currents of AC layer 2/3 pyramidal cells (Wang et al., 2006). Furthermore, the same group later showed that salicylate selectively and reversibly silences current-evoked activity of fast-spiking inhibitory interneurons, but not of regular spiking inhibitory or excitatory pyramidal neurons within AC (Su et al., 2009). Such a decrease in GABAergic inhibition in AC by the direct action of salicylate has been proposed to permit hyperactivity within this region following acute treatment of salicylate observed in earlier cytological studies (see Section "Auditory Cortex").

Effects of salicylate on GABAergic neurotransmission have also been observed outside of the classic central auditory pathway. Similar to the results in A1 described above, both evoked and spontaneous inhibitory postsynaptic currents were decreased in excitatory neurons in the CA1 region of the hippocampus (Gong et al., 2008). Similar observations were made in the dorsal horn of rat spinal dorsal horn neurons (Xu et al., 2005). These results indicate that, at least in vitro, salicylate, at the concentrations 
used (generally $1.4 \mathrm{mM}$ ), exerts its greatest effects on highly active inhibitory neurons.

Taken together, both in vitro and in vivo experiments indicate that acute dosing of salicylate generally depresses GABAergic neurotransmission in the brain, whereas compensatory mechanisms, such as increased GAD expression, are initiated following chronic dosing protocols. Such modulation of GABAergic neurotransmission would be expected to significantly alter spontaneous and stimulus-driven neural activity. However, as discussed next, the results from some electrophysiology experiments corroborate the salicylate-induced disinhibition hypothesis, whereas other experiments indicate that salicylate has more complex modulation on neurotransmission in the brain.

\section{ELECTROPHYSIOLOGY}

The consequences of salicylate treatment on sound-evoked and spontaneous firing rates have been measured in many regions of the mammalian auditory brain in vivo and/or in vitro (summarized in Figure 2). In this section, we will review results from electrophysiology studies of salicylate's effects on brain regions along the auditory pathway and compare them to results from noiseexposures designed to induce tinnitus. Finding brain regions which exhibit similar alterations in neural activity following salicylate treatment or noise trauma will likely yield a framework for understanding phantom sound perception in general.

\section{Cochlear nucleus}

As detailed above (see Section "Identifying Key Brain Regions"), following acute systemic salicylate treatment, c-fos expression was increased in $\mathrm{dCN}$, but not in $\mathrm{vCN}$. This result can be taken as evidence that acute salicylate treatment primarily affects nonlemniscal auditory structures. Indeed, activity in $\mathrm{vCN}$ tends to follow AN activity, whereas activity in $\mathrm{dCN}$ is more heavily modulated by non-auditory brain regions (Koerber et al., 1966). To the best of our knowledge electrophysiological recordings in $\mathrm{dCN}$ following salicylate treatment have only been carried out in brain slice preparations. Salicylate was found to either increase $(52.9 \%)$ or decrease $(47.1 \%)$ spontaneous firing rates of a subpopulation $(68 \%)$ of neurons in dCN (Basta et al., 2008). In a more recent patch clamp study, spontaneous and current-evoked firing rates of fusiform cells, but not cartwheel cells, in the dCN were selectively suppressed by salicylate (Wei et al., 2010). Furthermore, inhibitory postsynaptic currents recorded in fusiform cells of the $\mathrm{dCN}$ were significantly decreased by salicylate. These results indicated that suppressed firing rates in fusiform cells were most likely due to salicylate's direct effects on the intrinsic properties of this cell type and not the result of increased inhibition (Wei et al., 2010).

Significantly elevated spontaneous firing rates have been recorded from dCN fusiform cells in hamsters (Kaltenbach et al., 2004) and chinchillas (Brozoski et al., 2002) with behavioral evidence of tinnitus following exposure to loud tones. In subsequent studies by Brozoski and colleagues it was demonstrated that bilateral ablation of $\mathrm{dCN}$ in chinchillas with chronic tinnitus did not abolish tinnitus behavior in affected animals (Brozoski and Bauer, 2005); whereas ablation of $\mathrm{dCN}$ prior to noise trauma prevented the induction of tinnitus (Brozoski et al., 2012). While the dCN may play a role in the initial generation of tinnitus symptoms following intense sound exposure (Brozoski et al., 2012), the structure does not seem to be required to maintain the tinnitus percept (Brozoski and Bauer, 2005).

\section{Inferior colliculus}

Some of the earliest evidence that salicylate has direct effects on neural activity within the auditory brain was provided by electrophysiological recordings of altered neural activity in the IC following systemic delivery of salicylate. Following acute dosing in guinea pigs anesthetized with pentobarbital, spontaneous firing rates of a population of neurons in the eIC significantly increased (Jastreboff and Sasaki, 1986; Chen and Jastreboff, 1995), while the occurrence and duration of bursting type discharges in eIC increased for neurons sensitive to sound frequencies near the behaviorally assessed tinnitus pitch (Chen and Jastreboff, 1995). A mouse brain slice study observed similar frequency specific increases in spontaneous firing rates in cIC and eIC of neurons residing in anatomical regions receptive to sound frequencies at the animal's best hearing threshold (Basta and Ernst, 2004). Recordings from cIC of ketamine/xylazine anesthetized mice showed that acute salicylate treatment significantly decreased the occurrence of spontaneous bursting-type activity from single units as well as decreased the coefficient of variation for interspike intervals, indicating more regular spontaneous firing patterns (Ma et al., 2006). Furthermore, these changes were found to be greatest for single units with best frequencies below $27 \mathrm{kHz}$. Taken together, these electrophysiological results seem to corroborate observations of increased $c$-fos in eIC while the decreased spontaneous firing rates recorded in vivo agrees with the small decrease of $c$-fos observed in cIC following acute systemic treatment (Wallhausser-Franke, 1997).

Intravenous injection of lidocaine, a sodium channel blocker used to temporarily alleviate tinnitus in humans with chronic tinnitus (Perucca and Jackson, 1985; Reyes et al., 2002), reduced the salicylate-enhanced spontaneous firing rates of some guinea pig IC neurons for more than 30 minutes (Manabe et al., 1997). It is unclear from this study where in the IC these recordings were performed. We can speculate that since previous studies observed decreased spiking in cIC (Ma et al., 2006) and increased spiking in eIC (Chen and Jastreboff, 1995) that lidocaine may have been acting on neurons in eIC.

The amplitude of sound-evoked local field potentials (LFPs) recorded from the rat $\mathrm{IC}$ was unchanged following acute systemic salicylate injection; however, since the sound-evoked cochlear response (CAP) is decreased by salicylate treatment (see Section "Cochlear Effects"), the stable sound-evoked LFP amplitude of the IC indicates the neural signal was enhanced to a small degree in the brainstem (Sun et al., 2009). In the same study, application of sodium salicylate to the round window resulted in both decreased CAP amplitude and a correspondingly reduced sound-evoked LFP recorded from IC. These results provide further evidence that salicylate acts on the cochlea as well as directly on brain, most likely through the reduction of central inhibitory neurotransmission (see Section "Effects on GABAergic Neurotransmission”). 
Studies using the acute noise trauma model of tinnitus have observed increased spontaneous firing rates in the cIC near the exposure frequency after a week or more following noise exposure (Ma et al., 2006; Mulders and Robertson, 2009). Mulders and Robertson (2009) further demonstrated that inactivation, chemically, by cooling, or by complete ablation of the noise-exposed cochlea, reduced spontaneous firing rates of the contralateral cIC neurons compared to those of control animals. These results indicate that significantly enhanced spontaneous activity in cIC weeks after noise exposure still requires afferent input to exhibit these changes. While increased spontaneous activity weeks following noise trauma differs from acute salicylate treatment, where a decrease in c-fos was found in cIC (Wallhausser-Franke et al., 2003), chronic salicylate treatment similarly increased c-fos and 2-DG uptake in cIC (Wallhausser-Franke et al., 1996; Wu et al., 2003).

The role of the cIC in tinnitus may be different on short and long time scales. In acutely induced tinnitus from either noise trauma or salicylate, spontaneous firing rates in cIC do not increase immediately (salicylate or noise in mouse, Ma et al., 2006; noise in guinea pigs, Mulders and Robertson, 2009). In the case of noise trauma, spontaneous rates in the $\mathrm{dCN}$ and $\mathrm{cIC}$ increase starting 7 days or more after the exposure (Kaltenbach et al., 2004; Mulders and Robertson, 2009). Taken together, these results indicate that chronic, but not acute tinnitus may be associated with increased spontaneous firing in cIC.

One common feature between salicylate- and noise-induced tinnitus is the enhancement of the sound-evoked LFP recorded from the IC (Salvi et al., 1990; Mulders and Robertson, 2009; Sun et al., 2009). This enhanced response to sound may reflect a disinhibition in IC following manipulations that induce tinnitus. The precise location of this enhancement within the IC, (i.e., within cIC, dIC, or eIC), has yet to be determined.

\section{Medial geniculate body}

The only electrophysiological recordings of salicylate's effects on neural activity in MGB have been from extracellular recordings in vitro (Basta et al., 2008). In this study, the MGB (dorsal and ventral cells were analyzed together) exhibited the greatest proportion of neurons which had significantly altered spontaneous firing rates during salicylate application (dCN: 68.0\%; MGB: $80.8 \%$; AC: $71.4 \%$ of all recorded neurons); however, the direction of change in spontaneous firing rates of this MGB cell population were roughly equivalent ( $52.4 \%$ increase, $47.6 \%$ decrease).

We are unaware of any existing studies directly investigating the role of MGB in noise-induced tinnitus. Since MGB and the reticular nucleus of the thalamus play a critical role in gating auditory neural activity, these regions in concert with AC have been proposed to play a critical role in tinnitus perception (Llinas et al., 1999).

\section{Auditory cortex}

The effects of salicylate on spontaneous and sound-evoked neural activity within the auditory pathway have been most extensively studied in AC. The attention paid to this specific brain region may be warranted since several studies in animals have measured the largest changes in AC following acute systemic salicylate administration. Cytological studies indicate that metabolic activity in the core auditory cortex (A1 and AAF) was increased to the greatest extent over other auditory brain regions (Wallhausser-Franke et al., 2003, and see Section "Identifying Key Brain Regions"). The increased metabolic activity is presumed to reflect increased spontaneous neural activity; however, electrophysiology studies of AC do not seem to be in full agreement with this interpretation. In A1 and AAF, a small decrease in spontaneous firing rates has been measured following salicylate (Eggermont and Kenmochi, 1998; Yang et al., 2007; Lu et al., 2011), whereas a significant increase in secondary auditory cortex (A2) spontaneous firing rates was observed in anesthetized cats (Eggermont and Kenmochi, 1998). Since A1 and AAF receive projections primarily from the ascending lemniscal auditory pathway by way of vMGB (Huang and Winer, 2000), a small decrease observed in these regions may simply reflect the decreased cochlear output following salicylate treatment. In contrast, A2 is more heavily innervated by extralemniscal projections originating in $\mathrm{dMGB}$ (Huang and Winer, 2000). The increase in spontaneous neural activity in A2 may therefore indicate that the extralemniscal auditory structures play a more central role in salicylate-induced tinnitus. Furthermore, Eggermont and Kenmochi (1998) suggested that the presence of an incongruity in spontaneous firing rates across fields of AC may be an important feature distinguishing tinnitus-driven from acoustically driven sound perception.

The involvement of spontaneous activity in A1 during tinnitus induced by noise trauma has not been heavily investigated. Engineer et al. (2011) recorded sound-driven and spontaneous neural activity in A1 of rats 1 month following acute noise trauma. Rats with signs of tinnitus following noise exposure, assessed using the gap pre-pulse inhibition of the acoustic startle reflex paradigm, exhibited significantly increased spontaneous firing rates in $\mathrm{A} 1$; however, the reversal of signs of tinnitus using vagal-nerve stimulation paired with tones resulted in an additional increase in spontaneous firing rates. The authors conclude that increased spontaneous firing rates of neurons in A1 were not significantly correlated with evidence of tinnitus in rats.

In addition to simple spontaneous firing rates, other interesting changes in neural activity in AC have been observed following salicylate treatment. Ochi and Eggermont (1996) described changes in the temporal dynamics of spontaneous neural activity in A1 associated with salicylate treatment that may be more informative than simple statistics of population spontaneous firing rates. Salicylate was found to significantly increase the rebound to peak of the autocorrelation function, a mathematical tool used to look for repeating firing patterns of an individual neuron, indicating each spontaneous spike was followed by a prolonged interval before the next spike occurred. Furthermore, neurons in A1 were best modulated by click trains at slower presentation rates following salicylate. These changes in the temporal dynamics of A1 neural activity were hypothesized to be caused by increased afterhyperpolarization (AHP) resulting from enhanced activation of slow conducting $\mathrm{Ca}^{2+}$-activated $\mathrm{K}^{+}$-channels of cortical neurons (Ochi and Eggermont, 1996). The rationale for this hypothesis stems from studies of salicylate's action on peripheral nerve membranes at relatively high concentrations $(20 \mathrm{mM}$, Neto et al., 1980) compared to those levels measured in cerebrospinal fluid following systemic treatment $(1.4 \mathrm{mM}$, Jastreboff 
et al., 1986). In a more recent brain slice patch clamp study, a lower concentration of salicylate $(1.4 \mathrm{mM})$ failed to significantly alter AHP amplitude in AC neurons (Su et al., 2009). Additional in vivo studies with pharmacological manipulations are required to better understand the basis for the altered temporal dynamics observed in A1.

Sound-evoked activity in AC is greatly altered by systemically administered or locally applied salicylate (Lobarinas et al., 2006; Sun et al., 2009; Norena et al., 2010; Lu et al., 2011). Since the sound-evoked CAP is decreased in the cochlea by either route of delivery, the enhanced $\mathrm{AC}$ response indicates a signal gain increase occurs within the brain (Sun et al., 2009). Sun et al. (2009) recorded sound-evoked LFPs from electrodes chronically implanted over $\mathrm{AC}$ in awake and anesthetized rats before and after salicylate treatment. In awake rats, the amplitude of sound-evoked AC response was significantly enhanced following systemic dose of salicylate $(200 \mathrm{mg} / \mathrm{kg}$ i.p). Responses in AC were further enhanced in the same rats while under ketamine (NMDA receptor antagonist) anesthesia, whereas they were suppressed while under isoflurane $\left(\mathrm{GABA}_{\mathrm{A}}\right.$ receptor agonist) anesthesia. In a subsequent study, application of salicylate $(2 \mathrm{mM})$ to the brain surface over AC had similar effects on spontaneous (small decrease) and sound-evoked multiunit firing rates (significant increase) providing further evidence that these changes can be attributed to the direct effects of salicylate on the brain (Lu et al., 2011). Furthermore, co-application of the GABA transaminase inhibitor vigabatrin, which effectively increases the concentration of available GABA, or the $\mathrm{GABA}_{\mathrm{B}}$ receptor agonist baclofen to the brain surface reduced the salicylate-induced enhancement of sound-evoked multiunit firing rates (Lu et al., 2011).
Recently we showed that systemic salicylate treatment has significant and rapid effects on frequency receptive field tuning of neurons in the anesthetized rat A1 (Stolzberg et al., 2011). Following systemic administration of salicylate $(250$ or $300 \mathrm{mg} / \mathrm{kg}$ i.p.) the characteristic frequency, i.e., the sound frequency at which units were most sensitive, tended to shift toward $10-20 \mathrm{kHz}$ (Figure 3), frequencies which coincide with the pitch of salicylateinduced tinnitus determined from previous behavioral studies. We hypothesized that this dramatic change in the frequency sensitivity of neurons in A1 may be the result of two coincident effects of salicylate. First, salicylate significantly altered the profile of $\mathrm{OHC}$ sensitivity with the greatest reduction at low $(<10 \mathrm{kHz})$ and high $(>20 \mathrm{kHz})$ sound frequencies (in rat, Stolzberg et al., 2011). Additionally, salicylate had greater effects on high frequencies resulting in a somewhat sloping high-frequency hearing loss beginning at $16 \mathrm{kHz}$. Second, a decrease in cortical inhibition (Wang et al., 2006; Su et al., 2009; Lu et al., 2011) may result in shifting of neuronal characteristic frequencies on a rapid timescale. The overrepresentation of frequency along the tonotopic gradient of $\mathrm{A} 1$ has been observed following acoustic trauma (Irvine et al., 2000; Eggermont and Komiya, 2000), various learning paradigms using acoustic stimuli (Edeline et al., 1993; Rutkowski and Weinberger, 2005), and in humans with chronic tinnitus (Wienbruch et al., 2006). It has been proposed that an overrepresentation of a frequency along the tonotopic gradient in A1 may reflect the relative behavioral importance of a sound to the organism (Rutkowski and Weinberger, 2005).

In humans, a magnetoencephalography study by Wienbruch et al. (2006) observed a flattening of the tonotopic gradient and an enhanced dipole response using $40-\mathrm{Hz}$ auditory stimuli in A1
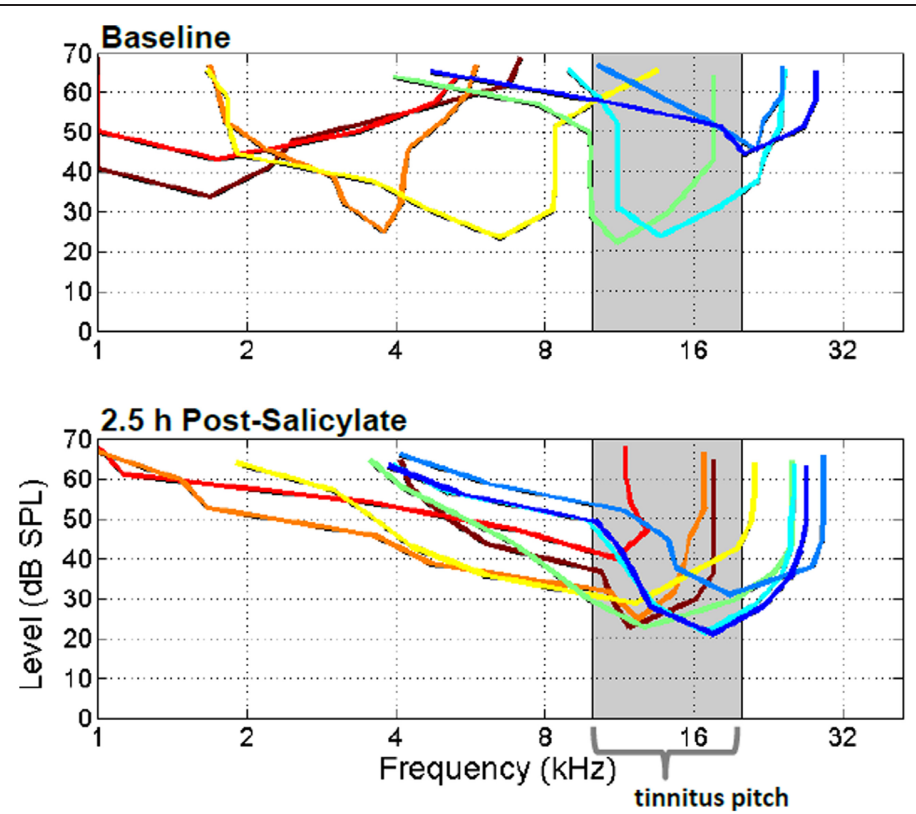

FIGURE 3 | Frequency tuning of eight simultaneously recorded extracellularly recorded multunits from primary auditory cortex before (top panel) and after systemic salicylate injection (bottom panel; $250 \mathrm{mg} / \mathbf{k g}$ i.p.). Following systemic salicylate treatment, the frequency tunings of tracked multiunits shifted maximal frequency sensitivities toward the $10-20 \mathrm{kHz}$ frequency region, near the estimated tinnitus pitch. Please see Stolzberg et al., 2011 for population statistics. [From Stolzberg et al. (2011) with permission]. 
of tinnitus subjects compared with controls. Recently, Engineer et al. (2011) demonstrated that the pairing of vagal nerve stimulation with tones was able to retune the distorted tonotopic gradient in A1 of rats with evidence of noise-induced tinnitus. The retuning of A1 tonotopy using the vagal nerve stimulation protocol reduced the number of rats with evidence of tinnitus using the gap-prepulse inhibition of the acoustic startle reflex test. The authors also found that distortion of the tonotopic gradient and broadening of tuning curves in A1 was significantly correlated with the degree of gap detection impairment (interpreted as the presence of tinnitus); however, changes in spontaneous firing rates or synchronization were not found to be correlated with the presence of tinnitus (Engineer et al., 2011).

Tonotopic overrepresentation in $\mathrm{A} 1$ has been proposed to result from the unmasking of normally inhibited intracortical connections (Eggermont and Roberts, 2004). Furthermore, the overrepresentation of frequencies in A1 results in an enhanced sound-evoked field potential. It remains uncertain whether or not such an overrepresentation is responsible for the generation of tinnitus signal or if it reflects persistent spontaneous neural activation somewhere else along the auditory pathway.

\section{DISCUSSION}

The salicylate toxicity model differs from the noise trauma model of tinnitus in several respects; however, a few aspects shared by the two models may facilitate the identification of key brain regions and patterns of neural activity involved in tinnitus perception in general.

\section{PERIPHERAL OR CENTRAL?}

One critical aspect to understanding the origins of tinnitus is whether a persistent signal is generated in the ear or abnormal patterns of neural activity occur within the brain. In humans with persistent subjective tinnitus, neural activity closely linked to changes in tinnitus loudness have been observed in AC, medial geniculate and other sites within the central nervous system (Lockwood et al., 1998; Muhlnickel et al., 1998; Lockwood et al., 2001; Reyes et al., 2002). Since salicylate is delivered to the brain and cochlea simultaneously following systemic treatment (Jastreboff et al., 1986) it is difficult to make a clear dissociation between the drug's peripheral and central effects. One of the primary sources of confusion regarding the locus of salicylate's generation of tinnitus signal, and the utility of the drug as a model for more common manifestations of tinnitus (i.e., following noise trauma or presbycusis), stems from the use of very high concentrations of sodium salicylate in AN fiber recordings.

Systemic treatment with sodium salicylate at moderate doses capable of inducing tinnitus either does not affect or slightly decreases spontaneous firing rates of AN fibers (Kumagai, 1992; Muller et al., 2003); however, at very high concentrations, salicylate begins to induce effects in the inner ear resulting in increased AN spontaneous firing rates (Evans and Borerwe, 1982; Ruel et al., 2008). Since behavioral evidence of tinnitus can be induced at the moderate doses of salicylate (Myers and Bernstein, 1965; Lobarinas et al., 2006), effects observed at very high concentrations of the drug may be difficult to directly relate to tinnitus generation or perception. Evidence from cytological studies which used acute salicylate treatment at moderate doses indicate that brain regions associated with the ascending lemniscal auditory system show either a decrease or no change in markers related to neural activity (Wallhausser-Franke et al., 2003). Since acoustic stimulation activates the lemniscal auditory pathway (Carretta et al., 1999), whereas acute systemic salicylate treatment at a moderate dose does not (Wallhausser-Franke, 1997; WallhausserFranke et al., 2003), it is unlikely that salicylate concentrations sufficient to induce tinnitus increase AN spontaneous firing rates. With regards to spontaneous AN activity, the use of a moderate dose of salicylate seems to be in agreement with other manipulations which induce decreased cochlear sensitivity, such as noise trauma (Liberman and Kiang, 1978) or selective inner hair cell loss (Wang et al., 1997). Taken together, it is likely that an acute moderate dose of salicylate induces tinnitus by alteration of neural activity within the brain and not inherited from increased spontaneous AN activity. Following salicylate, changes in cochlear sensitivity may, however, play a role in determining the pitch of tinnitus (Stolzberg et al., 2011).

\section{TIME COURSE OF SALICYLATE'S ACTIONS}

Existing research on the central effects of salicylate seems to be approximately split equally between acute and chronic dosing protocols. As discussed throughout this review, it is critical to consider the time course of effects following systemic salicylate treatment. Immediately following acute systemic treatment with salicylate, the extralemniscal pathway rapidly becomes hyperactive whereas spontaneous firing rates within lemniscal auditory structures remain relatively stable. On the other hand, chronic systemic salicylate treatment increases spontaneous firing rates within the lemniscal pathway (Wallhausser-Franke et al., 1996; Wu et al., 2003). These differential effects of acute and chronic salicylate treatment regimens are important to keep in mind while seeking putative mechanisms of salicylate-induced tinnitus. Studying the effects of acute and chronic salicylate treatment may yield information about various aspects of the drug's actions in the cochlea and the brain. Since tinnitus occurs rapidly after systemic administration of salicylate, the results from acute salicylate treatment in animals are likely to be highly informative about which brain regions are involved in the tinnitus perception per se, whereas the results from studying chronic salicylate treatment may be more informative regarding compensatory biochemical and neural mechanisms which may be targets for tuning out the tinnitus. One example of a compensatory mechanism is enhanced prestin expression and increased DPOAE response following chronic salicylate treatment (Huang et al., 2005; Yang et al., 2009; Chen et al., 2010).

\section{UTILITY OF SALICYLATE TOXICITY AS A MODEL FOR TINNITUS}

Induction of tinnitus using salicylate has a few important advantages over other methods of induction such as noise trauma. First, salicylate acts rapidly to induce tinnitus in subjects and is metabolized within days in most species, providing the ability to monitor physiological changes before, during, and after tinnitus. Second, the drug's effects on GABAergic neurotransmission may model other conditions which similarly decrease GABAergic neurotransmission associated with tinnitus in humans such 
as aging (Caspary et al., 1995, 1999; Ling et al., 2005). Third, salicylate can be administered to humans whereas intense noise trauma is often unethical (however see Ortmann et al., 2011). A fourth advantage is that the locus of drug delivery can be controlled. This provides a means to dissociate the drug's effects on various brain regions in vitro and in vivo. Finally, with proper dosing, salicylate can reliably induce tinnitus behavior in animals (Jastreboff et al., 1988a,b; Lobarinas et al., 2006), whereas noise trauma is highly variable at inducing tinnitus behavior in animals (Kraus et al., 2010).

There are also some important inherent limitations of the salicylate model of tinnitus. One such limitation of systemic administration of salicylate is that the drug induces binaural hearing loss and the perception of tinnitus is binaural. Unilateral noise trauma provides the opportunity to study tinnitus-related changes ipsilateral or contralateral to the exposed ear within individual brains. The reliability of tinnitus induction by salicylate may be considered a limitation of the model. The variable success of noise trauma in inducing tinnitus permits experimenters to identify potential mechanisms of susceptibility to tinnitus.

The utility of the salicylate toxicity model of tinnitus may ultimately reside in which of the drug's effects are similar with those of noise induced tinnitus. On an acute time course, identifying brain regions and abnormal patterns of neural activity coincident between multiple methods of tinnitus induction will likely yield a greater understanding of tinnitus perception in general. The entrenchment of the tinnitus signal in neural circuits on

\section{REFERENCES}

Axelsson, A., and Sandh, A. (1985). Tinnitus in noise-induced hearing loss. Br. J. Audiol. 19, 271-276.

Basta, D., and Ernst, A. (2004). Effects of salicylate on spontaneous activity in inferior colliculus brain slices. Neurosci. Res. 50, 237-243.

Basta, D., Goetze, R., and Ernst, A. (2008). Effects of salicylate application on the spontaneous activity in brain slices of the mouse cochlear nucleus, medial geniculate body and primary auditory cortex. Hear. Res. 240, 42-51.

Bauer, C. A., Brozoski, T. J., Holder, T. M., and Caspary, D. M. (2000). Effects of chronic salicylate on GABAergic activity in rat inferior colliculus. Hear. Res. 147, 175-182.

Bokemeyer, C., Berger, C. C., Hartmann, J. T., Kollmannsberger, C., Schmoll, H. J., Kuczyk, M. A., and Kanz, L. (1998). Analysis of risk factors for cisplatin-induced ototoxicity in patients with testicular cancer. Br. J. Cancer 77, 1355-1362.

Brozoski, T. J., and Bauer, C. A. (2005). The effect of dorsal cochlear nucleus ablation on tinnitus in rats. Hear. Res. 206, 227-236.

Brozoski, T. J., Bauer, C. A., and Caspary, D. M. (2002). Elevated fusiform cell activity in the dorsal

the timescale of years (for example see Schlee et al., 2008), however, may not be an approachable aspect of subjective tinnitus to investigate using the salicylate model.

\section{REMAINING QUESTIONS}

Some important experimental questions remain for tinnitus researchers which may be best approached using the salicylate toxicity model:

- Why does acute salicylate administration increase neural activity in extralemniscal, but not lemniscal auditory structures?

- Does pre-ablation of the dCN prevent salicylate-induced tinnitus (see Section "Cochlear Nucleus").

- What determines the behaviorally identified pitch of salicylateinduced tinnitus? Is it determined solely by salicylate's effects in the cochlea? Or is it determined by alterations of tonotopic gradients within the auditory brain?

- What do the differential effects of acute and chronic dosing of salicylate tell us about neuroplasticity and the entrenchment of the tinnitus signal?

\section{ACKNOWLEDGMENTS}

The authors thank S. Hayes for her valuable feedback on the manuscript. Work supported in part by grants from NIH (R01DC009091, R01DC009219, R03DC011374-01, F31DC010931), Tinnitus Research Consortium and Tinnitus Research Initiative.

by long-term salicylate treatment in the guinea pig: a plausible index of tinnitus. J. Neurophysiol. 80, 2113-2120.

Chen, G. D., and Jastreboff, P. J. (1995). Salicylate-induced abnormal activity in the inferior colliculus of rats. Hear. Res. 82, 158-178.

Chen, G. D., Kermany, M. H., D’Elia, A., Ralli, M., Tanaka, C., Bielefeld, E. C., Ding, D., Henderson, D., and Salvi, R. (2010). Too much of a good thing: long-term treatment with salicylate strengthens outer hair cell function but impairs auditory neural activity. Hear. Res. 265, 63-69.

Day, R. O., Graham, G. G., Bieri, D., Brown, M., Cairns, D., Harris, G., Hounsell, J., Platt-Hepworth, S., Reeve, R., Sambrook, P. N., and Smith, J. (1989). Concentrationresponse relationships for salicylateinduced ototoxicity in normal volunteers. Br. J. Clin. Pharmacol. 28, 695-702.

Didier, A., Miller, J. M., and Nuttall, A. L. (1993). The vascular component of sodium salicylate ototoxicity in the guinea pig. Hear. Res. 69, 199-206.

Douek, E. E., Dodson, H. C., and Bannister, L. H. (1983). The effects of sodium salicylate on the cochlea of guinea pigs. J. Laryngol. Otol. 97, 793-799.

Durrant, J., Wang, J., Ding, D., and Salvi, R. (1998). Are inner or outer hair cells the source of summating potentials recorded from the round window? J. Acoust. Soc. Am. 104, 370-377.

Edeline, J. M., Pham, P., and Weinberger, N. M. (1993). Rapid development of learning-induced receptive field plasticity in the auditory cortex. Behav. Neurosci. 107, 539-551.

Eggermont, J. J., and Kenmochi, M. (1998). Salicylate and quinine selectively increase spontaneous firing rates in secondary auditory cortex. Hear. Res. 117, 149-160.

Eggermont, J. J., and Komiya, H. (2000). Moderate noise trauma in juvenile cats results in profound cortical topographic map changes in adulthood. Hear. Res. 142, 89-101.

Eggermont, J. J., and Roberts, L. E. (2004). The neuroscience of tinnitus. Trends Neurosci. 27, 676-682.

Engineer, N. D., Riley, J. R., Seale, J. D., Vrana, W. A., Shetake, J. A., Sudanagunta, S. P., Borland, M. S., and Kilgard, M. P. (2011). Reversing pathological neural activity using targeted plasticity. Nature 470, 101-104. 
Evans, E. F., and Borerwe, T. A. (1982). Ototoxic effects of salicylates on the responses of single cochlear nerve fibres and on cochlear potentials. Br. J. Audiol. 16, 101-108.

Faye-Lund, H. (1985). The neocortical projection to the inferior colliculus in the albino rat. Anat. Embryol. 173, 53-70.

Fitzgerald, J. J., Robertson, D., and Johnstone, B. M. (1993). Effects of intra-cochlear perfusion of salicylates on cochlear microphonic and other auditory responses in the guinea pig. Hear. Res. 67, 147-156.

Gong, N., Zhang, M., Zhang, X. B., Chen, L., Sun, G. C., and Xu, T. L. (2008). The aspirin metabolite salicylate enhances neuronal excitation in rat hippocampal CA1 area through reducing GABAergic inhibition. Neuropharmacology 54, 454-463.

Gould, B. J., Huggins, A. K., and Smith, M. J. (1963). Effects of salicylate on glutamate dehydrogenase and glutamate decarboxylase. Biochem. J. 88, 346-349.

Gould, B. J., and Smith, M. J. (1965). Inhibition of rat brain glutamate decarboxylase activity by salicylate in vitro. J. Pharm. Pharmacol. 17, 15-18.

Graham, J. D., and Parker, W. A. (1948). The toxic manifestations of sodium salicylate therapy. Q. J. Med. 17, 153-163.

Guitton, M. J., Caston, J., Ruel, J., Johnson, R. M., Pujol, R., and Puel, J. L. (2003). Salicylate induces tinnitus through activation of cochlear NMDA receptors. J. Neurosci. 23, 3944-3952.

Guitton, M. J., Pujol, R., and Puel, J. L. (2005). m-Chlorophenylpiperazine exacerbates perception of salicylateinduced tinnitus in rats. Eur. J. Neurosci. 22, 2675-2678.

Halla, J. T., Atchison, S. L., and Hardin, J. G. (1991). Symptomatic salicylate ototoxicity: a useful indicator of serum salicylate concentration? Ann. Rheum. Dis. 50, 682-684.

Holt, A. G., Bissig, D., Mirza, N., Rajah, G., and Berkowitz, B. (2010). Evidence of key tinnitus-related brain regions documented by a unique combination of manganeseenhanced MRI and acoustic startle reflex testing. PloS One 5:e14260. doi: 10.1371/journal.pone.0014260

Huang, C. L., and Winer, J. A. (2000). Auditory thalamocortical projections in the cat: laminar and areal patterns of input. J. Comp. Neurol. 427, 302-331.

Huang, Z. W., Chen, P., Mei, L., Fan, Q. J., Xiao, B. K., and Wu, Z. Y. (2005). Differentially expressed gene profiles of cochlea in rats induced by acute and chronic sodium salicylate injection. Zhonghua Er Bi Yan Hou Tou Jing Wai Ke Za Zhi 40, 27-32.

Irvine, D. R., Rajan, R., and McDermott, H. J. (2000). Injuryinduced reorganization in adult auditory cortex and its perceptual consequences. Hear. Res. 147, 188-199.

Janssen, T., Boege, P., Oestreicher, E., and Arnold, W. (2000). Tinnitus and 2f1-f2 distortion product otoacoustic emissions following salicylate overdose. J. Acoust. Soc. Am. 107, 1790-1792.

Jastreboff, P. J., Brennan, J. F., Coleman, J. K., and Sasaki, C. T. (1988a). Phantom auditory sensation in rats: an animal model for tinnitus. Behav. Neurosci. 102, 811-822.

Jastreboff, P. J., Brennan, J. F., and Sasaki, C. T. (1988b). An animal model for tinnitus. Laryngoscope 98 , 280-286.

Jastreboff, P. J., Hansen, R., Sasaki, P. G., and Sasaki, C. T. (1986). Differential uptake of salicylate in serum, cerebrospinal fluid, and perilymph. Arch. Otolaryngol. Head Neck Surg. 112, 1050-1053.

Jastreboff, P. J., and Sasaki, C. T. (1986). Salicylate-induced changes in spontaneous activity of single units in the inferior colliculus of the guinea pig. J. Acoust. Soc. Am. 80, 1384-1391.

Kakehata, S., and Santos-Sacchi, J. (1996). Effects of salicylate and lanthanides on outer hair cell motility and associated gating charge. J. Neurosci. 16, 4881-4889.

Kaltenbach, J. A., Zacharek, M. A., Zhang, J., and Frederick, S. (2004). Activity in the dorsal cochlear nucleus of hamsters previously tested for tinnitus following intense tone exposure. Neurosci. Lett. 355, 121-125.

Kauer, J. S., Nemitz, J. W., and Sasaki, C. T. (1982). Tinnitus aurium: factor fancy. Laryngoscope 92, 1401-1407.

Kenmochi, M., and Eggermont, J. J. (1997). Salicylate and quinine affect the central nervous system. Hear. Res. 113, 110-116.

Kizawa, K., Kitahara, T., Horii, A., Maekawa, C., Kuramasu, T., Kawashima, T., Nishiike, S., Doi, K., and Inohara, H. (2010). Behavioral assessment and identification of a molecular marker in a salicylate-induced tinnitus in rats. Neuroscience 165, 1323-1332.

Koerber, K. C., Pfeiffer, R. R., Warr, W. B., and Kiang, N. Y. (1966). Spontaneous spike discharges from single units in the cochlear nucleus after destruction of the cochlea. Exp. Neurol. 16, 119-130.

Kraus, K. S., Mitra, S., Jimenez, Z., Hinduja, S., Ding, D., Jiang, H., Gray, L., Lobarinas, E., Sun, W. and Salvi, R. J. (2010). Noise trauma impairs neurogenesis in the rat hippocampus. Neuroscience 167 , 1216-1226.

Kujawa, S. G., Fallon, M., and Bobbin, R. P. (1992). Intracochlear salicylate reduces low-intensity acoustic and cochlear microphonic distortion products. Hear. Res. 64, 73-80.

Kumagai, M. (1992). Effect of intravenous injection of aspirin on the cochlea. Hokkaido Igaku Zasshi 67, 216-233.

Liberman, M. C., and Kiang, N. Y. (1978). Acoustic trauma in cats. Cochlear pathology and auditorynerve activity. Acta Otolaryngol. Suppl. 358, 1-63.

Ling, L. L., Hughes, L. F., and Caspary, D. M. (2005). Age-related loss of the GABA synthetic enzyme glutamic acid decarboxylase in rat primary auditory cortex. Neuroscience 132, 1103-1113.

Llinas, R. R., Ribary, U., Jeanmonod, D., Kronberg, E., and Mitra, P. P. (1999). Thalamocortical dysrhythmia: a neurological and neuropsychiatric syndrome characterized by magnetoencephalography. Proc. Natl. Acad. Sci. U.S.A. 96, 15222-15227.

Lobarinas, E., Yang, G., Sun, W. Ding, D., Mirza, N., Dalby-Brown, W., Hilczmayer, E., Fitzgerald, S., Zhang, L., and Salvi, R. (2006). Salicylate- and quinine-induced tinnitus and effects of memantine. Acta Otolaryngol. Suppl. 556, 13-19.

Lockwood, A. H., Salvi, R. J., Coad, M. L., Towsley, M. L., Wack, D. S., and Murphy, B. W. (1998). The functional neuroanatomy of tinnitus: evidence for limbic system links and neural plasticity. Neurology 50, 114-120.

Lockwood, A. H., Wack, D. S., Burkard, R. F., Coad, M. L., Reyes, S. A., Arnold, S. A., and Salvi, R. J. (2001). The functional anatomy of gazeevoked tinnitus and sustained lateral gaze. Neurology 56, 472-480.

Lu, J., Lobarinas, E., Deng, A., Goodey, R., Stolzberg, D., Salvi, R. J., and Sun, W. (2011). GABAergic neural activity involved in salicylateinduced auditory cortex gain enhancement. Neuroscience 189, 187-198.

Ma, W. L., Hidaka, H., and May, B. J. (2006). Spontaneous activity in the inferior colliculus of $\mathrm{CBA} / \mathrm{J}$ mice after manipulations that induce tinnitus. Hear. Res. 212, 9-21.
Manabe, Y., Yoshida, S., Saito, H., and Oka, H. (1997). Effects of lidocaine on salicylate-induced discharge of neurons in the inferior colliculus of the guinea pig. Hear. Res. 103, 192-198.

McFadden, D., Plattsmier, H., and Pasanen, E. (1984). Aspirin-induced hearing loss as a model of sensorineural hearing loss. Hear. Res. 16, 251-311.

McFadden, D., and Plattsmier, H. S. (1984). Aspirin abolishes spontaneous oto-acoustic emissions. J. Acoust. Soc. Am. 76, 443-448.

Miller, B., Sarantis, M., Traynelis, S. F., and Attwell, D. (1992). Potentiation of NMDA receptor currents by arachidonic acid. Nature 355, 722-725.

Mongan, E., Kelly, P., Nies, K., Porter, W. W., and Paulus, H. E. (1973). Tinnitus as an indication of therapeutic serum salicylate levels. JAMA 226, 142-145.

Muhlnickel, W., Elbert, T., Taub, E., and Flor, H. (1998). Reorganization of auditory cortex in tinnitus. Proc. Natl. Acad. Sci. U.S.A. 95, 10340-10343.

Mulders, W. H., and Robertson, D. (2009). Hyperactivity in the auditory midbrain after acoustic trauma: dependence on cochlear activity. Neuroscience 164, 733-746.

Muller, M., Klinke, R., Arnold, W., and Oestreicher, E. (2003). Auditory nerve fibre responses to salicylate revisited. Hear. Res. 183, 37-43.

Myers, E. N., and Bernstein, J. M. (1965). Salicylate ototoxicity; a clinical and experimental study. Arch. Otolaryngol. 82, 483-493.

Neto, M. S., Carvalho, A. C., and Callestini, E. A. (1980). Treatment of mandibular osteomyelitis in a pregnant syphilitic. Report of a clinical case. Rev. Assoc. Paul. Cir. Dent. Reg. Aracatuba 1, 7-13.

Norena, A., Micheyl, C., CheryCroze, S., and Collet, L. (2002). Psychoacoustic characterization of the tinnitus spectrum: implications for the underlying mechanisms of tinnitus. Audiol. Neurootol. 7, 358-369.

Norena, A. J., Moffat, G., Blanc, J. L., Pezard, L., and Cazals, Y. (2010). Neural changes in the auditory cortex of awake guinea pigs after two tinnitus inducers: salicylate and acoustic trauma. Neuroscience 166, 1194-1209.

Ochi, K., and Eggermont, J. J. (1996). Effects of salicylate on neural activity in cat primary auditory cortex. Hear. Res. 95, 63-76.

Ochi, K., and Eggermont, J. J. (1997). Effects of quinine on neural activity 
in cat primary auditory cortex. Hear. Res. 105, 105-118.

Oliver, D., He, D. Z., Klocker, N., Ludwig, J., Schulte, U., Waldegger, S., Ruppersberg, J. P., Dallos, P., and Fakler, B. (2001). Intracellular anions as the voltage sensor of prestin, the outer hair cell motor protein. Science 292, 2340-2343.

Ortmann, M., Muller, N., Schlee, W., and Weisz, N. (2011). Rapid increases of gamma power in the auditory cortex following noise trauma in humans. Eur. J. Neurosci. 33, 568-575.

Paul, A. K., Lobarinas, E., Simmons, R., Wack, D., Luisi, J. C., Spernyak, J., Mazurchuk, R., Abdel-Nabi, H., and Salvi, R. (2009). Metabolic imaging of rat brain during pharmacologically-induced tinnitus. Neuroimage 44, 312-318.

Peng, B. G., Chen, S., and Lin, X. (2003). Aspirin selectively augmented $\quad N$-methyl-D-aspartate types of glutamate responses in cultured spiral ganglion neurons of mice. Neurosci. Lett. 343, 21-24.

Perucca, E., and Jackson, P. (1985). A controlled study of the suppression of tinnitus by lidocaine infusion: (relationship of therapeutic effect with serum lidocaine levels). J. Laryngol. Otol. 99, 657-661.

Puel, J. L., Bobbin, R. P., and Fallon, M. (1990). Salicylate, mefenamate, meclofenamate, and quinine on cochlear potentials. Otolaryngol. Head Neck Surg. 102, 66-73.

Rachel, J. D., Kaltenbach, J. A., and Janisse, J. (2002). Increases in spontaneous neural activity in the hamster dorsal cochlear nucleus following cisplatin treatment: a possible basis for cisplatin-induced tinnitus. Hear. Res. 164, 206-214.

Ralli, M., Lobarinas, E., Fetoni, A. R., Stolzberg, D., Paludetti, G., and Salvi, R. (2010). Comparison of salicylate- and quinine-induced tinnitus in rats: development, time course, and evaluation of audiologic correlates. Otol. Neurotol. 31, 823-831.

Rauschka, H., Aboul-Enein, F., Bauer, J., Nobis, H., Lassmann, H., and Schmidbauer, M. (2007). Acute cerebral white matter damage in lethal salicylate intoxication. Neurotoxicology 28, 33-37.

Reyes, S. A., Salvi, R. J., Burkard, R. F., Coad, M. L., Wack, D. S., Galantowicz, P. J., and Lockwood, A. H. (2002). Brain imaging of the effects of lidocaine on tinnitus. Hear. Res. 171, 43-50.
Ruel, J., Chabbert, C., Nouvian, R., Bendris, R., Eybalin, M., Leger C. L., Bourien, J., Mersel, M., and Puel, J. L. (2008). Salicylate enables cochlear arachidonic-acidsensitive NMDA receptor responses. J. Neurosci. 28, 7313-7323.

Rutkowski, R. G., and Weinberger, N. M. (2005). Encoding of learned importance of sound by magnitude of representational area in primary auditory cortex. Proc. Natl. Acad. Sci. U.S.A. 102, 13664-13669.

Salvi, R. J., Saunders, S. S., Gratton, M. A., Arehole, S., and Powers, N. (1990). Enhanced evoked response amplitudes in the inferior colliculus of the chinchilla following acoustic trauma. Hear. Res. 50, 245-257.

Schlee, W., Weisz, N., Bertrand, O., Hartmann, T., and Elbert, T. (2008). Using auditory steady state responses to outline the functional connectivity in the tinnitus brain. PloS One 3:e3720. doi: 10.1371/journal.pone.0003720

Shargorodsky, J., Curhan, G. C., and Farwell, W. R. (2010). Prevalence and characteristics of tinnitus among US adults. Am. J. Med. 123, 711-718.

Shehata, W. E., Brownell, W. E., and Dieler, R. (1991). Effects of salicylate on shape, electromotility and membrane characteristics of isolated outer hair cells from guinea pig cochlea. Acta Otolaryngol. 111, 707-718.

Silverstein, H., Bernstein, J. M., and Davies, D. G. (1967). Salicylate ototoxicity. A biochemical and electrophysiological study. Ann. Otol. Rhinol. Laryngol. 76, 118-128.

Stolzberg, D., Chen, G. D., Allman, B. L., and Salvi, R. J. (2011). Salicylate-induced peripheral auditory changes and tonotopic reorganization of auditory cortex. Neuroscience $180,157-164$.

Su, Y. Y., Luo, B., Wang, H. T., and Chen, L. (2009). Differential effects of sodium salicylate on currentevoked firing of pyramidal neurons and fast-spiking interneurons in slices of rat auditory cortex. Hear. Res. 253, 60-66.

Sun, W., Lu, J., Stolzberg, D., Gray, L., Deng, A., Lobarinas, E., and Salvi, R. J. (2009). Salicylate increases the gain of the central auditory system. Neuroscience 159, 325-334.

Vane, S. J. (1998). Differential inhibition of cyclooxygenase isoforms: an explanation of the action of NSAIDs. J. Clin. Rheumatol. 4 (Suppl. 5), S3-S10.
Wallhausser-Franke, E. (1997). Salicylate evokes c-fos expression in the brain stem: implications for tinnitus. Neuroreport 8, 725-728.

Wallhausser-Franke, E., Braun, S., and Langner, G. (1996). Salicylate alters 2-DG uptake in the auditory system: a model for tinnitus? Neuroreport 7 , 1585-1588.

Wallhausser-Franke, E., Mahlke, C., Oliva, R., Braun, S., Wenz, G., and Langner, G. (2003). Expression of c-fos in auditory and non-auditory brain regions of the gerbil after manipulations that induce tinnitus. Exp. Brain Res. 153, 649-654.

Walsh, B. T., Miller, J. B., Gacek, R R., and Kiang, N. Y. S. (1972). Spontaneous activity in the eighth cranial nerve of the cat. Int. J. Neurosci. 3, 221-235.

Wang, H. T., Luo, B., Zhou, K. Q., $\mathrm{Xu}$, T. L., and Chen, L. (2006). Sodium salicylate reduces inhibitory postsynaptic currents in neurons of rat auditory cortex. Hear. Res. 215 77-83.

Wang, J., Powers, N. L., Hofstetter, P., Trautwein, P., Ding, D., and Salvi, R. (1997). Effects of selective inner hair cell loss on auditory nerve fiber threshold, tuning and spontaneous and driven discharge rate. Hear. Res. 107, 67-82.

Wei, L., Ding, D., Sun, W., XuFriedman, M. A., and Salvi, R (2010). Effects of sodium salicylate on spontaneous and evoked spike rate in the dorsal cochlear nucleus. Hear. Res. 267, 54-60.

Weisz, N., Hartmann, T., Dohrmann, K., Schlee, W., and Norena, A. (2006). High-frequency tinnitus without hearing loss does not mean absence of deafferentation. Hear Res. 222, 108-114.

Wienbruch, C., Paul, I., Weisz, N. Elbert, T., and Roberts, L. E. (2006). Frequency organization of the $40-\mathrm{Hz}$ auditory steady-state response in normal hearing and in tinnitus. Neuroimage 33, 180-194.

Wier, C. C., Norton, S. J., and Kincaid, G. E. (1984). Spontaneous narrowband oto-acoustic signals emitted by human ears: a replication. J. Acoust. Soc. Am. 76, 1248-1250.

Wier, C. C., Pasanen, E. G., and McFadden, D. (1988). Partial dissociation of spontaneous otoacoustic emissions and distortion products during aspirin use in humans. J. Acoust. Soc. Am. 84 230-237.

Wilson, J. P. (1980). Evidence for a cochlear origin for acoustic reemissions, threshold fine-structure and tonal tinnitus. Hear. Res. 2, 233-252.

Wu, J. L., Chiu, T. W., and Poon, P. W. (2003). Differential changes in Fosimmunoreactivity at the auditory brainstem after chronic injections of salicylate in rats. Hear. Res. 176, 80-93.

$\mathrm{Xu}, \mathrm{H}$., Gong, N., Chen, L., and $\mathrm{Xu}$, T. L. (2005). Sodium salicylate reduces gamma aminobutyric acidinduced current in rat spinal dorsal horn neurons. Neuroreport 16 813-816.

Yang, G., Lobarinas, E., Zhang, L., Turner, J., Stolzberg, D., Salvi, R. and Sun, W. (2007). Salicylate induced tinnitus: behavioral measures and neural activity in auditory cortex of awake rats. Hear. Res. 226, 244-253.

Yang, K., Huang, Z. W., Liu, Z. Q., Xiao, B. K., and Peng, J. H. (2009). Long-term administration of salicylate enhances prestin expression in rat cochlea. Int. J. Audiol. 48, 18-23.

Zhang, X., Yang, P., Cao, Y., Qin, L., and Sato, Y. (2011). Salicylate induced neural changes in the primary auditory cortex of awake cats. Neuroscience 172, 232-245.

Zheng, J., Shen, W., He, D. Z., Long, K. B., Madison, L. D., and Dallos, P. (2000). Prestin is the motor protein of cochlear outer hair cells. Nature 405, 149-155.

Zurek, P. M. (1981). Spontaneous narrowband acoustic signals emitted by human ears. J. Acoust. Soc. Am. 69, 514-523.

Conflict of Interest Statement: The authors declare that the research was conducted in the absence of any commercial or financial relationships that could be construed as a potential conflict of interest.

Received: 27 December 2011; paper pending published: 19 January 2012; accepted: 04 April 2012; published online: 20 April 2012

Citation: Stolzberg D, Salvi RJ and Allman BL (2012) Salicylate toxicity model of tinnitus. Front. Syst. Neurosci. 6:28. doi: 10.3389/fnsys.2012.00028

Copyright () 2012 Stolzberg, Salvi and Allman. This is an open-access article distributed under the terms of the Creative Commons Attribution Non Commercial License, which permits non-commercial use, distribution, and reproduction in other forums, provided the original authors and source are credited. 